\title{
Uso da Metadona no Tratamento da Dor Neuropática Não-Oncológica. Relato de Casos *
}

\section{Methadone to Treat Non-Oncologic Neuropathic Pain. Case Reports} Jeane Pereira da Silva Juver ${ }^{1}$, Nubia Verçosa Figueiredo ${ }^{2}$, Louis Barrucand ${ }^{3}$, Mauricio de Assis Tostes ${ }^{4}$

\section{RESUMO}

Juver JPS, Figueiredo NV, Barrucand L, Tostes MA - Uso da Metadona no Tratamento da Dor Neuropática Não-Oncológica. Relato de Casos

JUSTIFICATIVA E OBJETIVOS: O relato inclui sete casos de pacientes com dor neuropática crônica não-oncológica que não haviam obtido resultados satisfatórios com os tratamentos clássicos com antidepressivos tricíclicos e anticonvulsivantes. O uso da metadona, opióide sintético, semelhante à morfina, por via oral, é referido como alternativa no tratamento da dor neuropática pela sua ação antagonista não-competitiva sobre os receptores NMDA. Este estudo tem como objetivo avaliar o uso da metadona no tratamento de pacientes portadores de dores crônicas de origem neuropática não-oncológica que não melhoraram com os tratamentos clássicos.

RELATO DOS CASOS: São apresentados sete casos nos quais a dose de metadona foi titulada em consultas subseqüentes e as variáveis escore da intensidade álgica segundo a escala de faces, efeitos colaterais e melhora da capacidade funcional foram analisados no $1^{\circ}, 7^{\circ}, 14^{\circ}, 30^{\circ}$ e $180^{\circ}$ dias após o início do uso da medicação. Houve redução do escore da intensidade álgica e a ocorrência de efeitos colaterais como constipação, sedação, náuseas, cefaléia e insônia. Não foram observados efeitos colaterais do tipo euforia, sudorese, mioclonias, retenção urinária, redução de libido e depressão respiratória.

CONCLUSÕES: Os pacientes apresentaram resposta que fol considerada satisfatória ao uso da medicação, em baixas doses, controle dos efeitos colaterais com medidas simples e melhora representativa da capacidade funcional. A inclusão da metadona, nas condições deste estudo, mostrou-se uma opção eficaz, segura

* Recebido do (Received from) Programa de Pós-Graduação em Cirurgia Geral - Setor Anestesiologia do Departamento de Cirurgia da Faculdade de Medicina (FM) da Universidade Federal do Rio de Janeiro (UFRJ)

1. Pós-Graduanda do Curso de Pós Graduação em Cirurgia Geral - Setor Anestesiologia - Nível Mestrado, da Faculdade de Medicina da UFRJ. Anestesiologista Responsável Técnica pelo Centro de Cuidados Paliativos do Serviço de Hematologia e Oncologia Clínica Hospital Municipal Cardoso Fontes

2. Professora Adjunta e Doutora em Medicina do Departamento de Cirurgia da FM/UFRJ. Coordenadora da Graduação e Pós-Graduação em Anestesiologia da FM/UFRJ. Anestesiologista e Responsável pelo Ambulatório de Avaliação Pré-Anestésica do Hospital Universitário Clementino Fraga Filho (HUCFF) FM/UFRJ. Certificado de Área de Atuação em Dor SBA-AMB

3. Professor Titular de Patologia do Departamento de Anatomia Patológica da FM/UFRJ

4. Médico do Instituto de Psiquiatria e do Serviço de Psicologia Médica e Saúde Mental do HUCFF/UFRJ. Doutor em Medicina pela UFRJ

Apresentado (Submitted) em 07 de dezembro de 2004

Aceito (Accepted) para publicação em 06 de abril de 2005

Endereço para correspondência (Correspondence to)

Dra. Jeane Pereira da Silva Juver

Rua Caricé, $n^{\circ} 285$ Ap 205 - Bancários - llha do Governador

21920-100 Rio de Janeiro, RJ

E-mail: jeanejuver@aol.com.br

(c) Sociedade Brasileira de Anestesiologia, 2005 e de baixo custo, para o tratamento das dores de origem neuropáticas não-oncológicas.

Unitermos: ANALGÉSICOS, Opióide: metadona; DOR, Crônica: neuropática, não-oncológica

\section{SUMMARY}

Juver JPS, Figueiredo NV, Barrucand L, Tostes MA - Methadone to Treat Non-Oncologic Neuropathic Pain. Case Reports

BACKGROUND AND OBJECTIVES: This report includes seven patients with chronic non-oncologic neuropathic pain who have not responded to classic approaches with tricyclic antidepressant and anticonvulsant drugs. Oral methadone, a synthetic opioid similar to morphine, is referred as alternative to treat neuropathic pain due to its non-competitive antagonist action on NMDA receptors. This study aimed at evaluating methadone to treat chronic non-oncologic neuropathic pain patients who have not responded to classic approaches.

CASE REPORTS: Seven cases in which methadone dose was titrated in subsequent visits are presented. Pain severity according to facial scale, side effects and improved functional capacity were evaluated 1, 7, 14, 30 and 180 days after treatment beginning. There has been decrease in pain severity and in the incidence of side effects, such as constipation, sedation, nausea, headache and insomnia. There has been no euphoria, sweating, myoclonia, urinary retention, decreased libido and respiratory depression.

CONCLUSIONS: Patients responded satisfactorily to low dose medication, side effects were controlled with simple measures and there has been significant functional capacity improvement. In the conditions of this study, methadone was an effective, safe and low-cost option to treat non-oncologic neuropathic pain.

Key Words: ANALGESICS, Opioid: methadone; PAIN, Chronic: neuropathic; non-oncologic

\section{INTRODUÇÃO}

A dor é um sintoma definido pela IASP (Associação InterAnacional para o Estudo da Dor) como uma experiência sensorial e emocional desagradável associada a uma lesão efetiva ou potencial dos tecidos ou descrita em termos de tal lesão ${ }^{1}$.

Do ponto de vista temporal a dor pode ser classificada em aguda e crônica . A dor crônica passa a ser considerada doença quando interfere na qualidade de vida do indivíduo necessitando, por este motivo, de tratamentos eficazes para evitar o desenvolvimento de seqüelas físicas (atrofia muscular, redução de força muscular, encurtamento e redução da elasticidade dos tendões e ligamentos), e psíquicas (alterações do sono, ansiedade e depressão). Diante deste fato o desenvolvimento de novas opções terapêuticas é considerado uma questão de humanidade.

Em apoio à clínica, para a maior compreensão do fenômeno e como justificativa para proposta de tratamento, os estudos 
de fisiopatologia classificam a dor, quanto a sua origem, em nociceptiva ou em neuropática ${ }^{1-3}$.

Nos quadros de dor neuropática ocorrem alterações plásticas neuronais que incluem aumento do número dos canais iônicos, alterações na permeabilidade e sua distribuição; modificações na resposta às catecolaminas e, finalmente, ativação dos receptores N-metil-D-aspartato (NMDA). Estes são receptores excitatórios, ativados pelo glutamato e ligados a um canal iônico de cálcio. São ativados após despolarizações repetidas do neurônio, não participando da transmissão normal ${ }^{4}$.

As medicações classicamente utilizadas no tratamento da dor de origem neuropática são os antidepressivos, os anticonvulsivantes ${ }^{5}$, além de bloqueios anestésicos, utilizados em situações específicas. No entanto, alguns pacientes não apresentam resposta satisfatória, ou porque não ocorre alívio da dor, ou porque não toleram os respectivos efeitos colaterais.

$\mathrm{Na}$ busca de alternativas, os estudos com os antagonistas dos receptores NMDA, mostraram que esses fármacos poderiam ser utilizados como alternativas promissoras, caso fossem controlados os efeitos colaterais secundários ao seu uso ${ }^{6}$.

Com base em estudos farmacológicos para o tratamento de dor neuropática moderada ou intensa foi utilizado um opióide sintético semelhante à morfina, denominado metadona, agonista dos receptores opióides, e com ação antagonista não-competitiva sobre os receptores $\mathrm{N}$-metil-D-aspartato (NMDA) ${ }^{7-10}$

Focalizando as características particulares do metabolismo da metadona, pode-se citar: ausência de metabólitos farmacologicamente ativos; tendência a manter seus efeitos após uso prolongado; biodisponibilidade e meia-vida de eliminação individualizada. A metadona possui uma extensa metabolização hepática pelo processo de N-demetilação, em metabólitos farmacologicamente inativos, que não se acumulam na insuficiência renal e não são removidos por hemodiálise. Ela atravessa livremente a barreira placentária, podendo causar síndrome de abstinência em recém-nascidos, quando as mães fazem uso da metadona na gravidez. Contudo, as concentrações encontradas no leite materno não comprometem a amamentação ${ }^{10}$.

O objetivo deste relato é avaliar a importância do uso da metadona no tratamento de pacientes portadores de dores crônicas de origem neuropática não-oncológica que não responderam efetivamente aos tratamentos clássicos.

\section{RELATO DOS CASOS}

Após aprovação pelo Comitê de Ética em Pesquisa da Instituição foram observados os efeitos terapêuticos da metadona em sete pacientes, dentre aqueles atendidos no Ambulatório do Programa de Clínica de Dor e Cuidados Paliativos da Saúde Comunitária Coletiva do Hospital Universitário Clementino Fraga Filho (HUCFF) da Faculdade de Medicina (FM) da Universidade Federal do Rio de Janeiro (UFRJ), através de um estudo prospectivo.
Para a avaliação subjetiva da intensidade álgica foi utilizada a escala unidimensional de faces (EF), na qual são atribuídos escores a cada representação facial de forma crescente. O zero corresponde à ausência de dor e 4 a pior dor imaginável (Figura 1), obedecendo os critérios da Organização Mundial de Saúde (OMS).

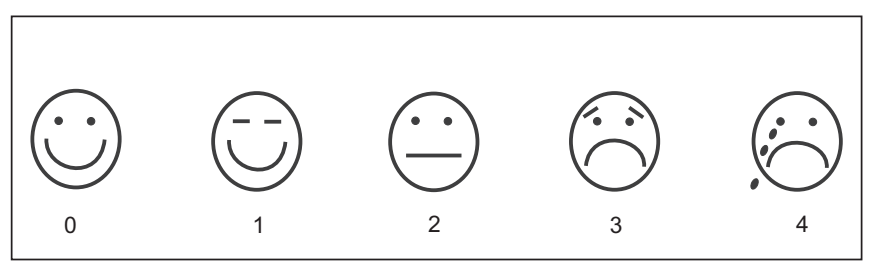

Figura 1 - Escala de Faces (EF)

escores: 0 - ausência de dor; 1 - dor leve; 2 - dor moderada; 3 -dor Intensa e 4 - dor insuportável

Os critérios de inclusão foram pacientes: 1) com dor de intensidade = a 2 na $E F ; 2$ ) dor de característica neuropática e não-oncológica; 3 ) que não tenham apresentado redução no mínimo $50 \%$ da intensidade álgica com os tratamentos clássicos; 4) que não apresentaram melhora da capacidade funcional ou que tenham apresentado efeitos colaterais intoleráveis com antidepressivos tricíclicos e/ou anticonvulsivantes; 5) maiores de 18 anos; 6 ) de ambos os sexos.

Os critérios de exclusão foram definidos com pacientes: 1) com alto grau de amplificação dolorosa; 2) grávidas ou amamentando; 3) que não apresentaram melhora funcional após o início do tratamento.

Após a primeira consulta foi titulada a dose da metadona até a redução da intensidade da dor, bem como a melhora funcional e dos efeitos colaterais. Estas variáveis foram avaliadas no sétimo $\left(7^{\circ}\right)$, décimo quarto $\left(14^{\circ}\right)$, trigésimo $\left(30^{\circ}\right)$ e centésimo octogésimo $\left(180^{\circ}\right)$ dias subseqüentes ao uso da medicação.

A melhora funcional foi analisada através dos seguintes parâmetros: autocuidado, atividades diárias laborativas e de reabilitação, humor e relacionamentos social, sexual e familiar.

Quanto aos efeitos adversos, caso ocorressem, os pacientes foram orientados sobre as atitudes a serem tomadas.

Na primeira consulta, a dose de metadona via oral foi titulada utilizando $2,5 \mathrm{mg}$ a cada 30 minutos até que fosse atingido escore 1 (dor leve) ou 0 (ausência de dor). No domicilio, caso a intensidade álgica atingisse o escore 2 (dor moderada), o paciente poderia utilizar a metadona na dose titulada na consulta com intervalo mínimo de 4 horas. A freqüência de administração da metadona e os parâmetros da capacidade funcional foram registrados na ficha de acompanhamento domiciliar padronizada pelo Programa de Clínica de Dor e Cuidados Paliativos (Quadro I), que deveria ser trazida em cada atendimento ambulatorial. No segundo atendimento, isto é $7^{\circ}$ dia, a dose da metadona foi calculada para duas administrações diárias. Foram relatados os efeitos colaterais e, quando necessário, foi iniciado tratamento específico. $\mathrm{Na}$ terceira avaliação, $14^{\circ} \mathrm{dia}$, foram anotados os escores da EF, 
Quadro 1 - Ficha de Acompanhamento Domiciliar

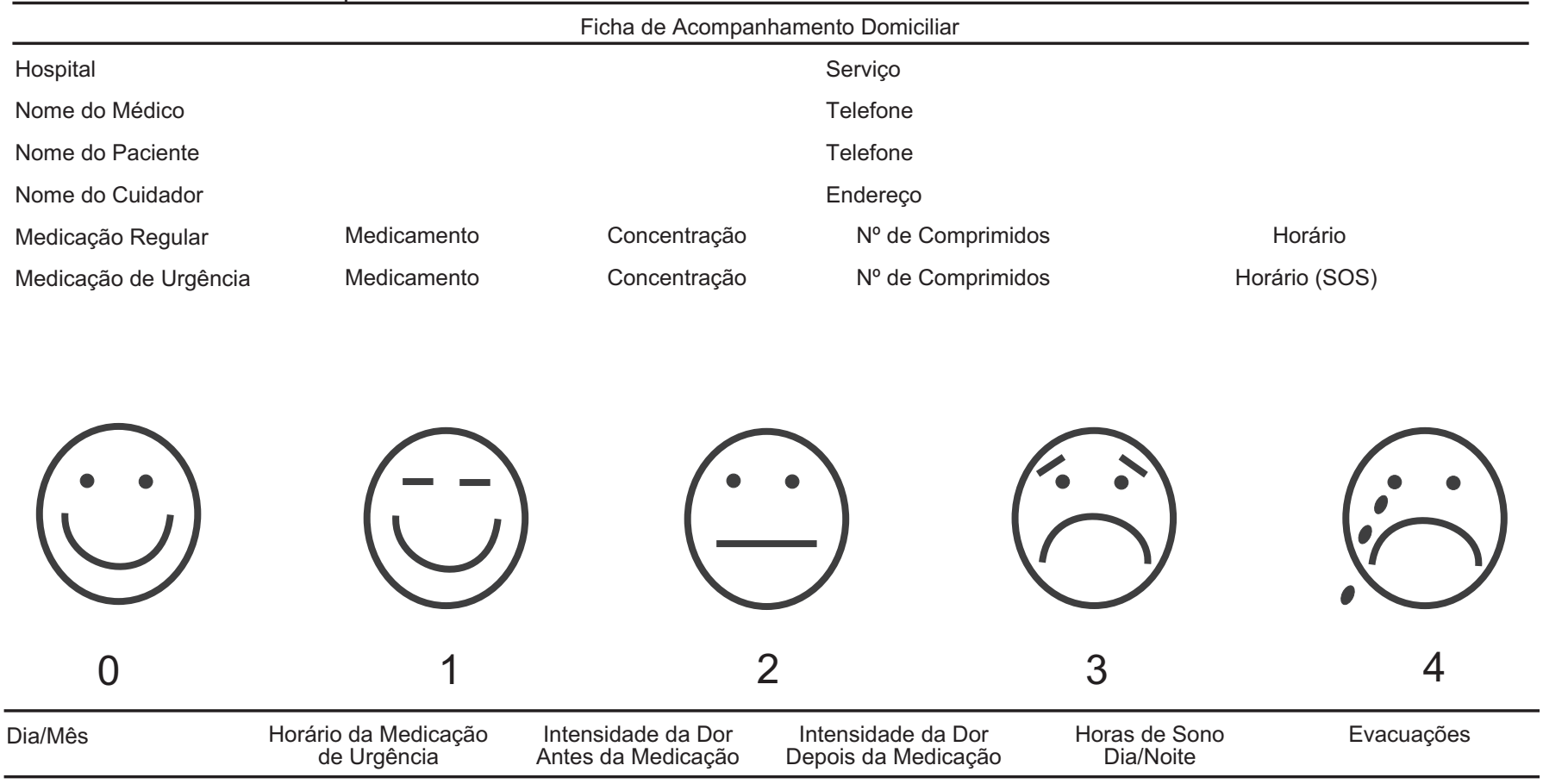

e o controle dos efeitos colaterais. Na quarta consulta, $30^{\circ}$ dia, foram reavaliados os dados do atendimento anterior, associados as análises de alterações da capacidade funcional dos pacientes. No quinto e último atendimento, $180^{\circ}$ dia, houve a avaliação da estabilização da dose utilizada e as alterações da capacidade funcional.

Caso 1 - Paciente do sexo feminino alegava ser portadora havia dois anos de dor no terceiro quirodáctilo à direita, em choque, de início espontâneo, recorrente de intensidade 3 na $E F$, além de hipoestesia de região tenar homolateral. No momento da consulta, encontrava-se em uso irregular de inibidor da COX-2 e carbamazepina sem alívio dos sintomas. Doméstica, após o diagnóstico da doença, foi licenciada pelo INSS. Desempenhava com dificuldade suas atividades diárias (cuidar da casa, lavar roupas, cozinhar). Residia sozinha, próximo a parentes com os quais recusava-se a manter contato por se considerar irritada em conseqüência da cirurgia e da dor que ainda a acompanhava. Raramente buscava atividades de lazer e evitava o convívio social em virtude de sua condição financeira e pela sua "incapacidade física". Mãe de filho adolescente, que reside com avó materna em sua cidade natal, e com quem mantém contatos eventuais. No momento não tinha parceiro sexual. Durante o tratamento procurou novo emprego. Melhorou a auto-estima, o convívio familiar e o social, dando início a planejamentos futuros. Evoluiu com controle do quadroálgico com doses estáveis de metadona (5 mg/dia). Efeito colateral principal foi sedação.

Caso 2 - Paciente do sexo masculino, com quadro de herpes zoster havia dois anos. Apresentava como seqüela, sintoma de dor em queimação na região inguinal, topografia de $L_{1}$ e $L_{2}$ à direita, do tipo contínua com períodos de exacerbação espontânea, de escore 2 na EF. Não havia relato de sintomas associados. Havia sido suspenso o uso de carbamazepina em decorrência de não haver tolerância aos efeitos colaterais. Os bloqueios analgésicos foram iniciados e posteriormente suspensos pela resposta insatisfatória. Aposentado que mantém atividades informais como carpinteiro para complementação de renda. Residia com esposa, com quem tem um bom relacionamento, mantendo vida sexual ativa. Pai de três filhos que residem próximos a sua residência e com os quais tem boa convivência. Relatou sentir-se triste e tinha necessidade de isolamento pelo caráter incapacitante da dor. Comentou que o retorno ao trabalho seria a solução para sua tristeza. Exprimiu vontade de solucionar o quadro álgico. Ao final do tratamento havia retornado ao trabalho informal, realizando pequenas tarefas. Referia preocupação com a possibilidade de realização de seus planos para o futuro, em virtude da crise existente no país quanto à possibilidade de trabaIho. O controle da dor foi obtido com doses diárias de $10 \mathrm{mg}$ de metadona. Referiu constipação intestinal durante o tratamento, que foi controlado com medicação laxante. Vol. 55, N 4, Julho - Agosto, 2005 
Caso 3 - Paciente do sexo feminino, havia 4 meses com quadro de dor de escore 4 na EF, contínua do tipo "doída" com episódios de dor em choque. Relatou redução da força muscular, sudorese e hiperemia, na topografia do nervo ulnar à esquerda, secundária a procedimento cirúrgico para correção de lesão dele no terço médio do antebraço, ocasionado por ferimento cortante. No momento da consulta, em uso irregular de inibidor da COX2 e paracetamol. Costureira, realizava pequenos trabalhos manuais para complementação de renda, porém sentia-se impossibilitada de realizá-los em conseqüência do quadro álgico. Separada do companheiro havia dez anos, residia com filha adolescente. Desempenhava com dificuldade suas atividades domésticas (lavar roupa, lavar louça), necessitando do auxílio da filha. Este fato a fazia sentir-se incapaz, acarretando muita tristeza em virtude de achar que esta "dependência" poderia prejudicar a vida social e sentimental de sua filha. Durante o tratamento, aceitou a sugestão de inclusão em sua rotina de exercícios fisioterápicos, e retornou ao mercado de trabalho informal. Neste caso, a redução da intensidade álgica foi atingida com 10 $\mathrm{mg} /$ dia de metadona. Apresentou durante sua evolução, quadro de constipação intestinal, sedação e cefaléia.

Caso 4 - Paciente do sexo feminino, portadora de HIV, referia ter dor nos membros inferiores havia dois anos, em peso, contínua, de escore 4 na EF, sem fatores de alívio ou agravamento, associada a parestesia, quadro que dificultava a deambulação. No momento da consulta estava em uso irregular de codeína associada a paracetamol e antirretrovirais. Paciente residia na casa dos pais, aposentada, evitava o convívio social por considerar sua doença difícil de lidar, ainda mais quando, além das modificações físicas, ocorriam sintomas dolorosos que comprometiam seu bom relacionamento com as demais pessoas. Relatava medo de não poder mais deambular em conseqüência da dor, fato que traria grande sofrimento por ser motivo de dependência de seus pais que considerava cansados de tanto sofrimento. No transcorrer do tratamento houve melhora do relacionamento familiar e social, ocasionando uma busca a atividades de lazer. A paciente alcançou alívio da dor com $10 \mathrm{mg} /$ dia de metadona. Náuseas e cefaléia foram referidas durante o tratamento.

Caso 5 - Paciente do sexo feminino apresentava havia um ano quadro de dor, do tipo "doída", contínua, de intensidade 3 na EF, que apresentava piora com a movimentação e melhorava com o repouso, associada a parestesia na borda ulnar do antebraço direito. Uso irregular de analgésicos e antiinflamatórios sem resolução do quadro. Paciente é técnica de enfermagem. Após cirurgias para tratamento de sinostose ulnar, foi licenciada pelo INSS. Residia com companheiro e fiIha maior. Os problemas conjugais se exacerbaram após as cirurgias e a sua saída do mercado de trabalho. O relato de sua doença e de seus problemas foram feitos com grande revolta. No momento da consulta, apesar de residir com seu companheiro, negava-se a ter vida sexual ativa. Na última consulta, encontrava-se fazendo acompanhamento fisiote- rápico. Fazia planos para o futuro a curto e médio prazo, buscava atividades de lazer em companhia da filha. Evoluiu com controle do quadro álgico com doses diárias de metadona $(20 \mathrm{mg})$. Apresentou quadro de constipação intestinal, controlada com laxante.

Caso 6 - Paciente do sexo masculino relatava havia oito anos quadro de dor em peso, contínua, difusa $\left(\mathrm{C}_{6}\right.$ a $\left.\mathrm{S}_{4}\right)$, com episódios de dor em choque, principalmente nos membros inferiores, de intensidade 2 na EF, com piora importante com a deambulação, associada a áreas de parestesia difusamente distribuídas e hiperestesia plantar bilateral. No momento da consulta não fazia uso de medicação para tratamento do quadro álgico, por ter suspendido por conta própria o uso de analgésicos, antidepressivos e anticonvulsivantes. Residia com esposa e dois filhos maiores, com os quais descreveu um bom relacionamento, havendo cooperação entre todos. Culpava a doença por todos os problemas de sua vida, pois no período que podia trabalhar e era detentor de bens, não era obrigado a conviver com os familiares de sua esposa. Em outros momentos referia-se à doença como punição por sua personalidade arrogante como patrão, marido e pai. Durante o tratamento iniciou caminhadas matinais sem exacerbação do quadro álgico após exercício. Mostrou-se mais disposto a tentar contornar os problemas familiares à medida que conseguiu maior autonomia. A redução da intensidade álgica foi atingida com $10 \mathrm{mg} / \mathrm{dia}$ de metadona. Apresentou insônia como efeito adverso.

Caso 7 - Paciente do sexo masculino relatou que há quatro anos tem dor de grande intensidade, 4 na EF, nos membros inferiores, em queimação, com episódio de dor em choque acompanhada de parestesia, hiperestesia e alodínia. Com a mobilização havia piora e melhorava com o repouso. Apresentava quadro semelhante nos membros superiores. Encontrava-se sem tratamento específico, após ter suspendido o uso de anticonvulsivantes e antidepressivos. Residia com companheira, com quem não tinha filhos por medo do caráter hereditário de sua doença, fato que ocasionava problemas conjugais. Negava ter vida sexual ativa. Evitava o convívio social pela característica estigmatizante de sua doença, principalmente em relação às manchas café-com-leite e pelo estrabismo. Relatou modificação dos hábitos de higiene pessoal por causa da alodínia, fato que dificultava o relacionamento social e interpessoal. Durante os atendimentos descreveu estar disposto a retornar à fisioterapia para reabilitação e readaptação ao mercado de trabalho. Retornou à atividade sexual e apresentou maior desenvoltura para realização de atividades de autocuidado. A dose diária de $20 \mathrm{mg}$ de metadona controlou o quadro álgico. Referiu náuseas durante o tratamento.

Os dados demográficos idade e sexo, evolução da dor e causas da dor estão representados na tabela I. Os escores de dor segundo a EF, antes da metadona (EFAM) e os atribuídos após titulação da metadona (EFATM), estão demonstrados na tabela II. A relação entre a dose utilizada da metadona e a 
ocorrência de efeitos colaterais como constipação, sedação, náuseas, cefaléia e insônia estão representadas na tabela III. Não foram observados efeitos colaterais do tipo euforia, sudorese, mioclonias, retenção urinária, redução de libido e depressão respiratória.

Tabela I - Dados Demográficos, Evolução da Dor e Causas da Dor

\begin{tabular}{|c|c|c|c|c|}
\hline Pacientes & Idade & Sexo & Evolução da Dor & Causas da Dor \\
\hline Caso 1 & 37 & Feminino & 2 anos & $\begin{array}{l}\text { Síndrome do túnel } \\
\text { do carpo }\end{array}$ \\
\hline Caso 2 & 71 & Masculino & 2 anos & Herpes zoster \\
\hline Caso 3 & 48 & Feminino & 4 meses & $\begin{array}{l}\text { Lesão do nervo ul- } \\
\text { nar }\end{array}$ \\
\hline Caso 4 & 40 & Feminino & 2 anos & $\begin{array}{l}\text { Polineuropatia } \\
\text { medicamentosal } \\
\text { HIV }\end{array}$ \\
\hline Caso 5 & 44 & Feminino & 1 ano & Sinostose ulnar \\
\hline Caso 6 & 53 & Masculino & 8 anos & $\begin{array}{l}\text { Síndrome de } \\
\text { Guillan Barrée }\end{array}$ \\
\hline Caso 7 & 46 & Masculino & 4 anos & $\begin{array}{l}\text { Neurofibromatose } \\
\text { tipo I }\end{array}$ \\
\hline
\end{tabular}

Tabela II - Escores de Dor Segundo a Escala de Faces durante o Estudo

\begin{tabular}{lcccccc}
\hline Pacientes & EFAM & EFATM & $7^{\circ}$ Dia & $14^{\circ}$ Dia & $30^{\circ}$ Dia & $180^{\circ}$ Dia \\
\hline Caso 1 & 3 & 1 & 0 & 0 & 0 & 0 \\
Caso 2 & 2 & 0 & 2 & 2 & 1 & 1 \\
Caso 3 & 4 & 1 & 2 & 2 & 2 & 2 \\
Caso 4 & 4 & 1 & 3 & 2 & 1 & 1 \\
Caso 5 & 3 & 1 & 2 & 2 & 1 & 1 \\
Caso 6 & 2 & 1 & 1 & 1 & 1 & 1 \\
Caso 7 & 3 & 1 & 1 & 0 & 0 & 0 \\
\hline
\end{tabular}

EFAM = escala de faces antes da metadona, EFATM = escala de faces após titulação de metadona

Tabela III - Dose de Metadona e Efeitos Colaterais

\begin{tabular}{lccl}
\hline Pacientes & DMT $(\mathrm{mg} / \mathrm{dose})$ & DMD $(\mathrm{mg} /$ dia $)$ & Efeitos Colaterais \\
\hline Caso 1 & 2,5 & 5 & Sedação \\
Caso 2 & 2,5 & 10 & Constipação \\
Caso 3 & 2,5 & 10 & $\begin{array}{l}\text { Constipação, sedação } \\
\text { e cefaléia }\end{array}$ \\
Caso 4 & 2,5 & 10 & Náuseas e cefaléia \\
Caso 5 & 5 & 20 & Constipação \\
Caso 6 & 5 & 10 & Insônia \\
Caso 7 & 5 & 20 & Náuseas \\
\hline
\end{tabular}

$\mathrm{DMT}=$ dose de metadona titulada, $\mathrm{DMD}=$ dose de metadona diária, dose média utilizada $12,14 \mathrm{mg} / \mathrm{dia}$

\section{DISCUSSÃO}

A avaliação da intensidade álgica foi feita através do relato do paciente, usando escalas que servem como parâmetro para quantificação da dose do analgésico e a respectiva complementação. A escala unidimensional de faces, que considera apenas a intensidade da dor, é de fácil entendimento, podendo ser utilizada por crianças e adultos ${ }^{11}$. É padronizada pelo Programa de Tratamento da Dor e Cuidados Paliativos do HUCFF.

Os pacientes foram avaliados por um período de 6 meses (180 dias), sendo que no primeiro mês as consultas eram realizadas no $1^{\circ}, 7^{\circ}, 14^{\circ}$ e $30^{\circ}$ dias. As avaliações clínicas eram mensais, porém os seus dados não foram considerados na análise dos resultados. Vale ressaltar que os pacientes continuaram sendo acompanhados pelos profissionais do Programa de Tratamento da Dor e Cuidados Paliativos da instituição, após o término do estudo.

Os casos apresentados mostraram redução de $50 \%$ ou mais da intensidade álgica durante o período do tratamento após o uso da metadona, com doses que podem ser consideradas baixas. Adose média de 12,14 mg/dia (mínimo de 5 mg e máximo de 20 mg/dia) divididas em duas administrações diárias, é considerada uma dose baixa com base nos dados encontrados na literatura onde as doses variam entre 60 e $80 \mathrm{mg} / \mathrm{dia}$ divididas em três ou quatro administrações de $20 \mathrm{mg}{ }^{12}$.

Os efeitos colaterais apresentados foram tolerados ou tratados com medicação adjuvantes. Em alguns casos, houve a resolução ou redução da sua intensidade, gerando desta forma um não comprometimento da qualidade de vida. Tal comportamento pode ser atribuído à titulação da dose de opióide a ser utilizada ${ }^{13}$.

O efeito colateral mais comum atribuído à ação dos opióides é a constipação intestinal, sendo considerada como obrigatória a prescrição de dieta e medicação laxante, concomitante à sua prescrição. Entre os pacientes observados não foi adotada tal postura. Nos casos em que tal efeito ocorreu, houve resolução do sintoma apenas com o uso da medicação adjuvante, extrato de sene e associações $\left(\right.$ Tamarine $^{\circledR}$ ), comportamento também aceito e descrito na literatura ${ }^{14}$.

Os estudos científicos evidenciam a menor freqüência do aparecimento de náusea e sedação com o uso de metadona quando comparado ao uso da morfina ${ }^{10}$. Neste grupo de pacientes estes efeitos também ocorreram, porém não houve necessidade de tratamento específico. O desenvolvimento de tolerância a esses efeitos pode ser observado com a continuidade do tratamento.

A insônia pode ser verificada em $10 \%$ a $20 \%$ dos pacientes que utilizam a metadona como fármaco de manutenção ${ }^{15}$. A mesma freqüência foi descrita nos casos relatados.

Apesar de ter sido constatada cefaléia em dois pacientes após o uso da metadona, não foi encontrado, na bibliografia consultada, nenhum relato semelhante.

Ao se analisar a resposta aos opióides quanto à melhora da capacidade funcional, observou-se excelentes resultados nos sete pacientes. Não foram utilizados escores para aferição desta variável. A afirmação deste parâmetro foi baseada nos relatos das modificações nos relacionamentos sociais, sexual, no trabalho, com os familiares, no retorno às atividades de reabilitação, na realização de atividades diárias com maior desenvoltura e na estruturação de planos futuros em curto e médio prazos. 
A perspectiva de novos tratamentos, entre os já existentes, revela-se como uma boa opção, ao permitir que os pacientes obtenham alívio de suas dores. Os resultados mostram a diversidade das doenças que podem apresentar quadros de dor de origem neuropática. A possibilidade de tratamento desses pacientes entre as várias especialidades médicas, reforça a necessidade da realização de pesquisas e trabaIhos que elucidem alguns detalhes da fisiopatologia da dor, que ainda permanecem obscuros. A inclusão da metadona pode ser uma alternativa de baixo custo com vistas à diminuição dos sintomas dolorosos de origem neuropática não-oncológica.

\section{Methadone to Treat Non-Oncologic Neuropathic Pain. Case Reports}

Jeane Pereira da Silva Juver, M.D.; Nubia Verçosa Figueiredo, M.D.; Louis Barrucand, M.D.; Mauricio de Assis Tostes, M.D.

\section{INTRODUCTION}

Pain is defined by IASP (International Association for the Study of Pain) as a disagreeable sensory and emotional experience associated to effective or potential tissue injury or described in such terms ${ }^{1}$.

Pain may be classified in acute and chronic ${ }^{1}$. Chronic pain is considered a disease when interfering with quality of life, thus requiring effective treatment to prevent the development of physical (muscle atrophy, decreased muscle strength, shortening and decreased elasticity of tendons and ligaments) or psychical (sleep disorders, anxiety and depression) sequelae. Due to these facts, the development of new treatment options is a matter of humaneness.

To support the clinic in understanding this phenomenon and as a justification for a treatment proposal, pathophysiology studies have classified the origin of pain as nociceptive or neuropathic ${ }^{1-3}$.

Neuropathic pain leads to plastic neuronal changes including increased number of ion channels, changes in their patency and distribution; abnormal responses to catecholamines and $\mathrm{N}$-methyl-D-aspartate (NMDA) receptors activation. These are excitatory receptors activated by glutamate and bound to a calcium ion channel. They are activated after repeated neuronal depolarizations and do not participate on normal transmission ${ }^{4}$.

Classic drugs to treat neuropathic pain are antidepressants and anticonvulsants ${ }^{5}$ in addition to anesthetic blocks in specific situations. However, some patients do not respond to treatment, either because there is no pain relief or because they do not tolerate side effects.
In the search for alternatives, studies with NMDA receptor antagonists have shown that they could be promising alternatives provided their side effects were controlled ${ }^{6}$.

Based on pharmacological studies for the treatment of moderate to severe neuropathic pain, a synthetic opioid similar to morphine, namely methadone, which is an opioid receptor agonist with non-competitive antagonist action on NMDA receptors, was used ${ }^{7-10}$.

Some unique characteristics of methadone metabolism are: lack of pharmacologically active metabolites; trend to maintain its effects after prolonged use; bioavailability and individualized excretion half-life. Methadone is extensively metabolized by the liver through the $\mathrm{N}$-demethylation process into pharmacologically inactive metabolites which do not build up during renal failure and are not removed by hemodialysis. It crosses placental barrier freely and may cause withdrawal syndrome in neonates when mothers are given methadone during pregnancy. However, concentrations found in breast milk do not prevent breastfeeding ${ }^{10}$.

This report aimed at evaluating the importance of methadone to treat chronic non-oncologic neuropathic pain patients who have not responded to classic approaches.

\section{CASE REPORTS}

After the Institution's Research Ethics Committee approval, the therapeutic effects of methadone were observed in seven patients from the Ambulatory of Pain Treatment and Paliative Care, Collective Community Health Program, Hospital Universitário Clementino Fraga Filho (HUCFF), School of Medicine (FM) Universidade Federal, Rio de Janeiro (UFRJ), through a prospective study.

For subjective evaluation of pain severity, the one-dimension facial scale (FS) was used, in which scores are attributed to each face representation in ascending order. Zero corresponds to no pain and 4 to the worst possible pain (Figure 1), according to World Health Organization (WHO) criteria.

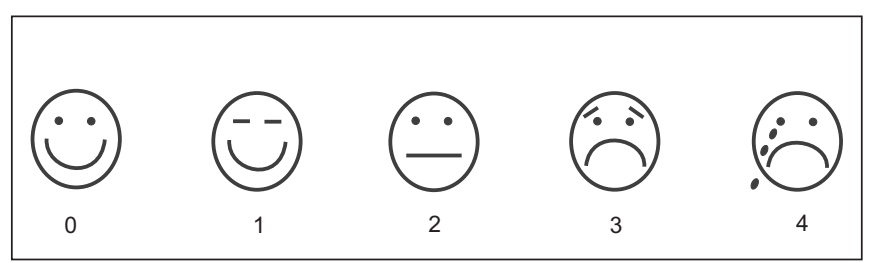

Figure 1 - Facial Scale (FS)

scores: 0 - no pain; 1 - mild pain; 2 - moderate pain; 3 - severe pain; 4 - unbearable pain

Inclusion criteria were patients: 1 ) with pain severity $\geq 2$ in FS; 2) non-oncologic neuropathic pain; 3 ) who have not improved at least $50 \%$ with classic approaches; 4 ) who have not improved their functional capacity or who could not tolerate side effects of tricyclic antidepressants and/or anticonvulsants; 5) aged above 18 years; 6 ) of both genders. 
Exclusion criteria were patients: 1) with high degree of pain amplification; 2) pregnant or breastfeeding; 3) without functional improvement after beginning of treatment.

Methadone dose was titrated after the first visit until pain severity was decreased and functional and side effects were improved. These variables were evaluated $7,14,30$ and 180 days after treatment beginning.

Functional improvement was evaluated by the following parameters: self-care, daily working and rehabilitation activities, mood, and social, sexual and family relationships.

Patients were told what to do in case of adverse effects.

Oral methadone dose was titrated in the first visit using 2.5 mg methadone every 30 minutes until score 1 (mild pain) or 0 (no pain) was reached. At home, if pain intensity reached score 2 (moderate pain), patients could use methadone dose titrated during visit with minimum intervals of 4 hours.

Frequency of methadone administration and functional capacity parameters were recorded in the home follow up record standardized by the Pain Treatment and Palliative Care Program (Chart I), which should be taken by patients to every ambulatory visit.

During the second visit, that is, seven days after, methadone dose was calculated for two daily administrations. Side effects were reported and specific treatment was started when needed. At third evaluation ( $14^{\text {th }}$ day), previous visit data were re-evaluated and associated to patients' functional ca- pacity changes. In the last visit $\left(180^{\text {th }}\right.$ day), dose stabilization and functional capacity changes were evaluated.

Case 1 - Female patient referring pain in the third right finger in shock, with spontaneous onset, recurrent and FS score 3 , in addition to hypoesthesia in the ipsilateral tenar region. At evaluation, patient was under irregular use of COX2 inhibitor and carbamazepine without symptoms relief. Domestic servant, patient was licensed by Social Security after diagnosis. Patient would carry out daily activities with difficulty (take care of home, washing, cooking). Patient lives alone, close to relatives with whom she refused to have contact for considering herself irritated as a consequence of the surgery and of the pain that still persisted. Patient would seldom look for entertainment and avoided social contact due to her financial condition and "physical disability". Patient is mother of a male adolescent who lives with the maternal grandmother in her hometown with whom she maintains sporadic contact. Currently she had no sexual partner. During treatment she looked for a new job, improved her self-esteem, family and social contact and started planning for the future. Patient evolved with pain control with stable methadone doses (5 $\mathrm{mg} /$ day). Major side effect was sedation.

Case 2 - Male patient with herpes zoster for two years, complaining of continuous burning pain in the inguinal region, topography of $L_{1}$ and $L_{2}$ to the right, with periods of spontaneous

\section{Chart I - Home Follow-up Record}

\begin{tabular}{|c|c|c|c|c|}
\hline \multicolumn{5}{|c|}{ Home Follow-Up Record } \\
\hline Hospital & & & Service & \\
\hline Physician's name & & & Telephone & \\
\hline Patient's name & & & Telephone & \\
\hline Caretaker's name & & & Address & \\
\hline Regular Medication & Drug & Concentration & $\mathrm{N}^{0}$ of tablets & Time \\
\hline Urgency Medication & Drug & Concentration & $\mathrm{N}^{\circ}$ of tablets & Time (SOS) \\
\hline
\end{tabular}

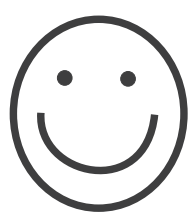

0

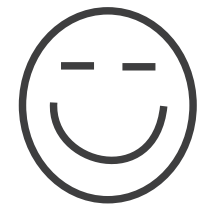

1

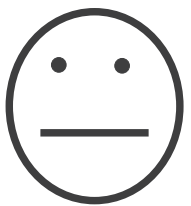

2

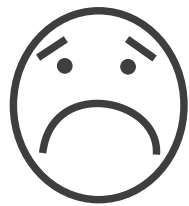

3

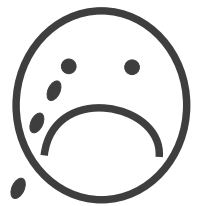

4

\begin{tabular}{lccc}
\hline Day/ Month & $\begin{array}{c}\text { Time of Urgency } \\
\text { Medication }\end{array}$ & Pain Intensity & Pain Intensity \\
BFTER medication & Hours of sleep & Evacuations \\
\hline
\end{tabular}


exacerbation and FS score 2. There were no reports on associated symptoms. Carbamazepine had been withdrawn due to intolerance to side effects. Analgesic blocks were started and then interrupted due to unsatisfactory response. Patient is retired with informal carpenter activities to complement income. Patient lives with his wife with whom he has good relations and active sexual life. Patient has three sons who live nearby and with whom he has good relationships. Patient reported being unhappy and needing to be by himself due to disabling pain. He mentioned that going back to work would be the solution for his unhappiness and that he was willing to solve his pain problem. At the end of the treatment he had returned to informal activities performing minor tasks. He was concerned with meeting his future goals due to the employment crisis the country was going through. Pain was controlled with daily $10 \mathrm{mg}$ methadone. Patient referred intestinal constipation during treatment. This adverse effect was controlled with laxatives.

Case 3 - Female patient with continuous and "painful" FS score 4 pain for 4 months, with episodes of shock pain. Patient has reported muscle strength decrease, sweating and hyperemia, at ulnar nerve topography to the left, secondary to surgical procedure to correct ulnar nerve lesion at forearm medium third, caused by cutting wound. At evaluation, patient was under irregular use of COX2 inhibitor and paracetamol. Dressmaker, she would do minor manual works to complement income however she had to stop them due to pain. Separated from the companion for ten years she lived with the adolescent daughter. Patient had difficulties to perform domestic activities (washing, cleaning) needing her daughter's help. This made her feel disabled and brought lots of unhappiness for feeling that this "dependence" could impair her daughter's social and sentimental life. During treatment she accepted the suggestion of including physical therapy exercises to her daily life and has returned to informal work. In this case, pain was controlled with $20 \mathrm{mg} /$ day methadone. Side effects were intestinal constipation, sedation and headache.

Case 4 - Female HIV patient referring lower limbs continuous, pain, at weight, FS score 4 for two years, without relieving or worsening factors, associated to paresthesia which would impair ambulation. At evaluation she was under irregular use of codeine associated to paracetamol and anti-retroviral drugs. Patient lived with parents, was retired and avoided social contact for considering that her disease was difficult to deal with, moreover when, in addition to physical changes, there were painful symptoms compromising her good relationship with other people. Patient reported fear of no longer being able to walk as a consequence of pain, fact which would bring lots of suffering because she would depend on her parents whom she considered tired of so much suffering. During treatment there has been improved family and social relationship leading to the search for leisure activities. Pain was relieved with $10 \mathrm{mg} /$ day methadone. Side effects were nausea and headache.
Case 5 - Female patient with "painful", continuous, FS score 3 pain, which would worsen with movements and improve at rest, associated to paresthesia of right forearm ulnar edge. Patient was under irregular use of analgesic and anti-inflammatory drugs without resolution. Patient is a nurse. After surgeries to treat ulnar synostosis, patient was licensed by Social Security. Patient lived with her companion and her daughter. Matrimonial problems were exacerbated after surgeries and her quitting her job. Patient reported her disease and problems with rage. At evaluation, although living with her companion, she denied having active sexual life. During last visit she was under physiotherapic follow up, had medium and long-term plans and was looking for leisure activities together with the daughter. Pain was controlled with daily methadone doses $(20 \mathrm{mg})$. Side effect was intestinal constipation controlled with laxatives.

Case 6 - Male patient with continuous, at weight, diffuse $\left(C_{6}\right.$ to $\mathrm{S}_{4}$ ), FS score 2 pain with episodes of shock pain especially on lower limbs, with major worsening with ambulation and associated to diffusely distributed paresthesia and bilateral plantar hyperesthesia. At evaluation patient was not taking medication because he had withdrawn at his own judgment the use of analgesics, antidepressants and anticonvulsants. Patient lived with wife and two adult children with whom he reported good relationships and cooperation among all. Patient blamed the disease for all his problems because when he was able to work and had money he was not forced to be with his wife's relatives. In other moments he would refer to the disease as punishment to his arrogant personality as boss, husband and father. During treatment he started morning walks without pain exacerbation after exercising. He was more prone to try to control family problems as acquired more autonomy. Pain was decreased with $10 \mathrm{mg} /$ day methadone and side effect was insomnia.

Case 7 - Male patient with lower limbs burning pain, FS score 4 , for four years, with episodes of shock pain followed by paresthesia, hyperesthesia and allodynia. Pain would worsen with movements and improve at rest. Patient had similar symptoms in upper limbs. Patient was under no specific treatment after having withdrawn anticonvulsants and antidepressants. Patient lived with his companion with whom he had no children by fear of his disease being hereditary, fact that caused matrimonial problems. Patient denied having active sexual life and avoided social contact due to the stigmatizing character of his disease, especially coffee and milk stains and strabismus. Patient reported changes in personal hygiene habits due to allodynia, which would further impair his social and interpersonal relationships. During treatment patient reported being willing to return to physical therapy to readapt to the marketplace. Patient has returned to sexual activities and improved his self-care activities. Pain was controlled with $20 \mathrm{mg} /$ day methadone and reported side effect was nausea during the treatment. 
Age, gender, pain evolution and causes are shown in table I. FS pain scores before methadone (FSBM) and after methadone (FSAM) are shown in table II. Relationship between methadone doses and side effects, such as constipation, sedation, nausea, headache and insomnia are shown in table III. There were no euphoria, sweating, myoclonia, urinary retention, decreased libido and respiratory depression.

Table l-Demographics Data, Pain Evolution and Pain Causes

\begin{tabular}{lcccl}
\hline Patients & Age & Gender & $\begin{array}{c}\text { Pain } \\
\text { Evolution }\end{array}$ & Pain Causes \\
\hline Case 1 & 37 & Female & 2 years & Carpal tunnel syndrome \\
Case 2 & 71 & Male & 2 years & Herpes zoster \\
Case 3 & 48 & Female & 4 months & Ulnar nerve injury \\
Case 4 & 40 & Female & 2 years & Drug polineuropathy /HIV \\
Case 5 & 44 & Female & 1 year & Ulnar synostosis \\
Case 6 & 53 & Male & 8 years & Guillan Barrée syndrome \\
Case 7 & 46 & Male & 4 years & Neurofibromatosis type I \\
\hline
\end{tabular}

Table II - Pain Scores According to Facial Scale throughout the Study

\begin{tabular}{lcccccc}
\hline Patients & FSBM & FSAM & $7^{\text {th }}$ Day & $14^{\text {th }}$ Day & $30^{\text {th }}$ Day & $180^{\text {th }}$ Day \\
\hline Case 1 & 3 & 1 & 0 & 0 & 0 & 0 \\
Case 2 & 2 & 0 & 2 & 2 & 1 & 1 \\
Case 3 & 4 & 1 & 2 & 2 & 2 & 2 \\
Case 4 & 4 & 1 & 3 & 2 & 1 & 1 \\
Case 5 & 3 & 1 & 2 & 2 & 1 & 1 \\
Case 6 & 2 & 1 & 1 & 1 & 1 & 1 \\
Case 7 & 3 & 1 & 1 & 0 & 0 & 0 \\
\hline
\end{tabular}

FSBM = facial scale before methadone, FSAM = facial scale after methadone titration

Table III - Methadone Dose and Side Effects

\begin{tabular}{lccl}
\hline Patients & MTD (mg/dose) & MDD (mg/day) & Side Effects \\
\hline Case 1 & 2,5 & 5 & Sedation \\
Case 2 & 2,5 & 10 & Constipation \\
Case 3 & 2,5 & 10 & $\begin{array}{l}\text { Constipation, seda- } \\
\text { tion and headache }\end{array}$ \\
Case 4 & 2,5 & 10 & $\begin{array}{l}\text { Nausea and head- } \\
\text { ache }\end{array}$ \\
Case 5 & 5 & 20 & Constipation \\
Case 6 & 5 & 10 & Insomnia \\
Case 7 & 5 & 20 & Nausea \\
\hline
\end{tabular}

MTD = methadone titrated dose, $\mathrm{MDD}=$ methadone daily dose, mean dose $=12.14 \mathrm{mg} / \mathrm{day}$

\section{DISCUSSION}

Pain severity was evaluated through patients' reports using scales which act as parameters for analgesic dose quantification and respective complementation. The one-dimension facial scale, considering pain severity alone, is easy to understand and may be used for children and adults ${ }^{11}$. It was standardized by the Pain Treatment and Palliative Care Program, HUCFF.

Patients were evaluated in regular 30-day intervals throughout the study. Data obtained at 30 days were the $4^{\text {th }}$ evaluation and values obtained at 180 days were the $5^{\text {th }}$ evaluation. It is worth stressing that patients continued to be followed by professionals of the Pain Treatment and Palliative Care Program after study completion.

Our cases have shown $50 \%$ or more decrease in pain severity during treatment with methadone, with doses considered low. Mean $12.14 \mathrm{mg} /$ day dose (minimum of $5 \mathrm{mg}$ and maximum of $20 \mathrm{mg} /$ day) divided in two daily doses, is considered a low dose according to data in the literature where doses vary from 60 to $80 \mathrm{mg} /$ day divided in three to four $20 \mathrm{mg} \mathrm{doses}^{12}$.

Side effects were tolerated or treated with adjuvant drugs. There has been resolution or decreased severity in some cases, thus not impairing quality of life. Such behavior may be attributed to titration of the opioid dose ${ }^{13}$.

Most common opioid side effect is intestinal constipation being mandatory the simultaneous prescription of diet and laxative medication. This posture was not adopted with our patients. In the presence of such effect, symptoms were resolved with adjuvant drugs, senna extract and associations (Tamarine ${ }^{\circledR}$ ), which is a described and accepted approach ${ }^{14}$. Scientific studies have observed lower incidence of nausea and sedation with methadone as compared to morphine ${ }^{10}$. This effect has been also observed in our group, however with no need for specific treatment. Tolerance to these effects may be observed with prolonged treatment.

Insomnia may be observed in $10 \%$ to $20 \%$ of patients using methadone as maintenance drug ${ }^{15}$. The same frequency was observed in our cases.

Although there has been headache referred by two patients after methadone, no similar report was found in our references.

In analyzing improved functional capacity after opioids, excellent results were observed in all patients. No scores were used to evaluate this variable. This statement was based on reports on changes in social, sexual, family and work relationships, on the return to rehabilitation activities, on easily performing daily activities and on short and long term plans.

The possibility of new therapies is seen as a major option for pain relief. Results have shown the diversity of diseases that may be followed by neuropathic pain. The possibility of treating these patients throughout different medical specialties reinforces the need for researches and studies to clarify the details of its physiopathology, which still remain unexplained. Methadone may be a low cost option to treat non-oncologyc pain. 


\section{REFERÊNCIAS - REFERENCES}

01. Gozzani JL - Fisiopatologia da Dor, em: Cavalcanti IL, Madalena ML - Dor, $1^{\text {a }}$ Ed, Rio de Janeiro, Editora SAERJ , 2003;13-35.

02. Teixeira MJ, Teixeira WGI, Kraychette DC - Epidemiologia Geral da Dor, em: Teixeira MJ - Dor: Contexto Interdisciplinar, $1^{\mathrm{a}} \mathrm{Ed}$, Curitiba, Editora Maio, 2003;53-63.

03. Bonica JJ, Loeser JD - History of Pain Concepts and Therapies, em: Loeser JD - Bonica's Management of Pain - $3^{\text {rd }}$ Ed, Philadelphia: Williams \& Wilkins, 2001;3-16.

04. Drummond JP - Neurofisiologia, em: Drummond JP - Dor Aguda: Fisiopatologia, Clínica e Terapêutica, São Paulo, Editora Atheneu, 2000;1-25.

05. Galer BS, Dworkin RH - Pharmacologic Treatment of Neuropathic Pain, em: Galer BS, Dworkin RH - A Clinical Guide to Neuropathic Pain, $1^{\text {st }}$ Ed, New York, Editora The Mc Graw-Hill Companies, 2000;49-51.

06. Ebert B, Thorkildsen C, Andersen S et al - Opioid analgesics as non-competitive $\mathrm{N}$-methyl-D-aspartate (NMDA) antagonists. Biochem Pharmacol, 1998;56:553-559.

07. Portenoy RK, Foley KM - Chronic use of opioids analgesics in non-malignant pain: report of 38 cases. Pain, 1986;25:171-186.

08. Gorman AL, Elliott KJ, Inturrisi CE - The d- and I- isomers of methadone bind to the non-competitive site on the $\mathrm{N}$-methyl-d-aspartate (NMDA) receptor in rat forebrain and spinal cord. Neurosci Lett, 1997;223:5-8.

09. Eriksen J, Sjogren P - Opioids in Pain Management. Acta Anaesthesiol Scand, 2001;41:1-3.

10. Ribeiro S, Schimidt AP, Schimidt SRG - O uso de opióides no tratamento da dor crônica não-oncológica: o papel da metadona. Rev Bras de Anestesiol, 2002;27:644-651.

11. Sakata RK, Hisatugo MK, Aoki SS et al - Avaliação da Dor, em: Cavalcanti IL, Madalena ML - Dor, $1^{\text {a }}$ Ed, Rio de Janeiro, Editora SAERJ, 2003;53-94.

12. Dal Pan GJ, Mc Arthur JC - Diagnosis and management of sensory neuropathies in HIV infection. Aids Clinical Care, 1994;6:9-13.

13. Portenoy RK, Foley KM, Inturrisi CE - The nature of opioids responssiveness for neuropathic pain: new hipotheses derived from studies of opioids infusions. Pain,1990;43:273-286.

14. Clark JD, Elliott J - A case of methadone-induced movement disorder. Clin J Pain, 2001;17:375-377.

15. Portenoy RK - Opioids therapy for chronic nonmalignant pain: a review of the critical issues. J Pain Symptom Manage, 1996;11: 203-213.

\section{RESUMEN}

Juver JPS, Figueiredo NV, Barrucand L, Tostes MA - Uso de la Metadona en el Tratamiento del Dolor Neuropático No Oncológico. Relato de Casos

JUSTIFICATIVA Y OBJETIVOS: EI relato incluye siete casos de pacientes con dolor neuropático crónico no oncológico, que no habían logrado resultados satisfactorios con los tratamientos clásicos con antidepresivos tricíclicos y anticonvulsivantes. El uso de la metadona, opioide sintético, semejante a la morfina, por vía oral, es referido como alternativa en el tratamiento del dolor neuropático por su acción antagonista no competitiva sobre los receptores NMDA. EI objetivo de este estudio es evaluar el uso de la metadona en el tratamiento de pacientes portadores de dolores crónicos de origen neuropático no oncológico, que no mejoraron con los tratamientos clásicos.

RELATO DE LOS CASOS: Se presentan siete casos en los cuales la dosis de metadona fue titulada en consultas subsiguientes y los variables resultados de la intensidad álgica según la escala de aspectos, efectos colaterales y mejora de la capacidad funcional fueron analizados en el $1^{\circ}, 7^{\circ}, 14^{\circ}, 30^{\circ}$ y $180^{\circ}$ días después del inicio del uso de la medicación. Hubo reducción de los resultados de la intensidad álgica y la ocurrencia de efectos colaterales como constipación, sedación, náuseas, cefalea e insomnio. No fueron observados efectos colaterales del tipo euforia, sudoresis, mioclonias, retención urinaria, reducción de la libido y depresión respiratoria

CONCLUSIONES: Los pacientes presentaron respuesta considerada satisfactoria al uso de la medicación, en bajas dosis; control de los efectos colaterales con medidas simples y mejora representativa de la capacidad funcional. La inclusión de la metadona, en las condiciones de este estudio, se mostró una opción eficaz, segura y de bajo valor, para el tratamiento de los dolores de origen neuropáticos no oncológicos. 